\title{
CLINICAL ENDPOINTS IN CLINICAL TRIALS OF CHEMORADIATION FOR ANAL CANCER
}

\author{
Glynne-Jones $\mathbf{R}^{\mathbf{1}}$, Adams $\mathbf{R}^{2}$, Lopes $\mathbf{A}^{3}$ Meadows $\mathbf{H}^{3}$. \\ ${ }^{1}$ Centre for Cancer Treatment, Mount Vernon Hospital, Northwood, Middlesex, UK; ${ }^{2}$ Cardiff \\ University and Velindre Cancer Centre, Cardiff, UK; ${ }^{3}$ Cancer Research UK \& UCL Cancer \\ Trials Centre, London, UK.
}

\section{Running title: "endpoints in anal cancer"}

\section{SUMMARY}

This review examines endpoint reporting in randomized controlled trials (RCTs) of radical chemoradiation for the treatment of squamous cell carcinoma of the anus (SCCA). The types, frequency, and definitions of clinical primary and secondary endpoints, and patient-reported outcome measures (PROMs) reported in the methods and results sections of papers (and protocols if available) were examined. Only 6 published RCTs (2877 patients) were identified. The primary outcome measures varied across the trials analysed: 2 used diseasefree survival (DFS); 1 Progression-Free Survival; 2 local failure; 1 colostomy-free survival. The definition for these terms was not consistent between trials - particularly for treatment failure (local, regional, and distant). Secondary endpoints include overall survival, complete clinical response, quality of life, toxicity and compliance. The quality of outcome reporting in RCTs of SCCA is inconsistent. A core outcome set including clinical and PROMs with standardised definitions is needed to improve reporting of randomised trials examining definitive chemoradiation treatment for SCCA.

\section{0 words}

KEY WORDS: squamous cell carcinoma of the anus, outcomes, endpoints, chemoradiation, clinical trial. 


\section{Introduction}

Phase III randomised controlled trials (RCT) are considered the best design to assess the efficacy of a particular intervention in clinical medicine - based on clinically meaningful and statistically significant impact on patient outcomes. In addition, safety and efficacy are used to determine whether a treatment is worth using in clinical practice. Outcome measures such as overall survival, disease-free survival, objective response or stable disease, or improvements in specific symptoms are balanced against toxicity, loss of function, risk of second malignancy or death. In most Phase III cancer trials, overall survival, which is a clear and unequivocal event, is considered the benchmark endpoint. Surrogate early endpoints such as pathological complete response (pCR) or clinical complete response (cCR) are often useful within clinical trials for cancer using chemoradiation (CRT) because they represent a more rapidly attained assessment of treatment effects. A recent overview elegantly addresses clinical trial endpoints and design in general. ${ }^{1}$

Randomised trials of squamous cell carcinoma of the anus (SCCA) have used multiple timeto-event endpoints with different disease-related and survival events, but a standardised definition of the outcome measures is lacking. SCCA has a low rate of distant metastases unless recurrence occurs at the primary site. Hence, local control is the primary aim and radical chemoradiation (CRT) is the mainstay of treatment, which is associated with significant acute and late morbidity.

In analysing ACT II trial data ${ }^{2}$, the present authors have discovered many pitfalls in terms of the primary and secondary endpoints used in this trial and their definitions when comparing results with other trials. For example, The ACT II trial protocol used 3 primary endpoints, the complete response rate, recurrence-free survival and acute toxicity. Patients who have had a complete local excision (as a result of biopsy or removal of a non-suspicious nodule and usually T1NO) were ineligible. However, patients with local excision and involved margins were eligible. If disease has been macroscopically resected and there is no evidence of residual or nodal disease on imaging, CCR is an inappropriate primary endpoint, and recurrence-free survival should be used. In retrospect therefore some endpoints were inappropriate for the eligibility.

Definitions appear to be inconsistent between the different phase III trials. For example the definition of local failure may include or exclude disease at sites within the pelvis caused by 
loco-regional invasion ie inguinal nodes and occasionally includes new separate tumours that arise independently in the same area.

The International Rare Cancers Initiative (IRCI) aims to increase international collaboration in clinical trials, and has developed a trial for metastatic/relapsed $\mathrm{SCCA}^{\mathbf{3}}$. If successful, further multicentre international studies will be undertaken but one of the barriers is the variably defined outcomes, which require tight standardisation as recommended by both The International Conference on Harmonisation (ICH) guidelines ${ }^{4}$ (ICH) and the CONSORT statement $^{4}$. Endpoint consistency has been previously discussed for squamous cell cancers of the head and neck (SCCHN $)^{\mathbf{5}}$ but not for SCCA. Consistent and unambiguous definitions of time-to-event endpoints in recurrence has also been highlighted as an issue for more common cancers, and the need to avoid changes to the pre-specified primary and secondary outcome (endpoint) measures after the trial has started ${ }^{\mathbf{6}}$.

Our aim in this review was to evaluate the standard phase III endpoints, and produce recommendations for their definition and use in future trials of SCCA.

\section{LITERATURE SEARCH}

A computerised literature search examined relevant English literature using Pubmed, Medline and Cancerlit over the period 1974 to December 2015, supplemented by hand-searching of abstracts from recent international meetings. We employed the key words - anal cancer, squamous cell carcinoma, local recurrence, survival, concurrent irradiation, chemotherapy, radiotherapy (RT), chemoradiation (CRT), combined modality (Online Table 1 includes a full list of terms). Studies were eligible if they met the following criteria: patients with SCCA had been randomly allocated, or treatment had been prospectively determined. Of the 2139 records identified through database searching, screening of the titles resulted in the exclusion of irrelevant or duplicate publications. Of the 76 abstracts or full-text articles assessed for eligibility, 11 publications of 6 randomized relevant trials were found. Only papers published in English were reviewed. We read the full text of 148 articles, which seemed likely to offer original and relevant information to the scope of this review on the defined endpoints of complete clinical response, loco-regional control (LRC), disease-free survival (DFS), progression-free survival (PFS) relapse-free survival (RFS), colostomy-free survival (CFS), cause-specific (CSS) and overall survival (OS). 


\section{RESULTS}

Only six phase III randomised controlled trials (RCTs) and updates have been published in SCCA over the past 25 years ${ }^{\mathbf{2}, \mathbf{8}-15}$. We examined the primary and secondary endpoints and their interpretation. Table 1 shows their primary/secondary endpoints and Table 2 how the composite disease-related endpoints are variably defined.

\section{Overall Survival (time to death from any cause) and cause-specific survival (time to cancer-specific death )}

Overall survival is a clearly defined endpoint, which is not modified by investigator definitions of failure, compliance of patients with long-term follow-up, clinical or radiographic assessments, or physician bias. However, the mortality rate is relatively low in anal cancer and there are competing risks for death in an elderly population ${ }^{\mathbf{1 6}}$. To evaluate whether a treatment improves survival, a large number of patients or long term follow-up would be needed in order to test a realistic effect size. Also, the availability of subsequent effective surgical salvage treatment in that location, and the effect of successive treatment lines with novel chemotherapy or biological systemic therapy, potentially introduce bias because they can prolong survival. In addition, the risk of non-cancer deaths from the intervention rises with increasing time.

In ACT I ${ }^{9}$ and ACT II ${ }^{2}$, only 77\% (182/236) and 73\% (155/211) deaths respectively were due to anal cancer and recent surgical reports suggest abdominoperineal resection for nonmetastatic recurrent or persistent anal cancer can salvage some recurrences, resulting in 5 years OS around $60 \%{ }^{17,18}$. These factors can substantially dilute the observed treatment effect on survival and explain why, despite large differences in local control, in RCT's comparing radiation alone with chemoradiotherapy, initial treatment did not impact on OS ${ }^{\mathbf{9 , 1 0 , 1 3}}$. However significant differences in ACT I were observed using cause-specific survival (CSS) as an endpoint (only anal-cancer-related deaths). The disadvantages of this endpoint is the potential for misclassification of causes of death and varying practice in the inclusion or exclusion of treatment-related death as an event.

\section{Other composite time-to-event endpoints}

Other cancer related time-to-event endpoints include a disease-related event such as progression or disease recurrence and survival depending on whether all patients have disease 
which is detectable or not at the time of randomisation.

Major differences exist in the radiotherapy treatment schedules (planning volumes and doses), not only between but also within the individual RCTs, partly because of a reliance on early response - either histopathological ${ }^{\mathbf{8}}$ or clinical $^{\mathbf{1 1}, 12}$ to decide the appropriate total radiation dose after the first phase of treatment. Also varying compliance with the planned treatment as defined by protocolised dose reductions of chemotherapy for toxicity, may impact on these results.

\subsection{Event-free survival (EFS)}

EFS is not an immediately meaningful term for clinicians unless the event or various events of interest are well defined and not excessively complex. The RTOG9811 trial protocol and recent phase I/II studies ${ }^{19}$ define EFS as the time from date of registration to the date of death from any cause, first evidence of disease progression, evaluated as non-complete response $(\mathrm{nCR})$ at the second evaluation after $\mathrm{CR}$, undergoing colostomy or first evidence of second primary cancer, whichever happens first.

\subsection{Disease free Survival (DFS)}

DFS serves both as a surrogate endpoint and as an endpoint in itself ${ }^{\mathbf{2 0}}$. DFS is defined as time from randomisation to first event of recurrent disease or death (occasionally persistent/progressive disease and/or second primary tumours are counted as events). The RTOG 9811 trial $^{11}$ used DFS as the primary endpoint (which included second malignancies). Unless pre-specified, the date of disease recurrence is subject to measurement error and other forms of bias, because of differences in the precision and timing of clinical follow-up, and radiological and histological assessments between arms. Standardized follow-up protocols may therefore be required.

DFS often counts the following as an event: nCR (timed 4-11 weeks following CRT see above), radiological local, nodal, pelvic or distant disease following a post CRT CR or death from any cause. However, it is paradoxical to consider this a meaningful endpoint for patients who have slow or no response to treatment, but are salvaged by surgery and thereafter have no clinical disease.

At randomisation all patients have measurable disease (clinically or radiologically). Following chemoradiation the majority become disease-free, but up to $10 \%$ will have 
persistent disease. This does not fit endpoints such as DFS (usually used following surgery when there is a time-point when there is definitely no detectable tumour). Analysis may be performed only on patients who are disease-free at a fixed time-point (e.g. 6 months posttreatment) when the use of DFS is appropriate, but important early information is lost. There is both an early and late pattern to loco-regional relapse /failure. There are also patients who are never free from disease. Such patients can be considered a treatment failure and we can assume that their event occurs at the time of randomisation. In contrast, for other patients there is a point usually 3-9 months following completion of treatment, when we conclude that the patient has residual active cancer and surgical salvage is required. This point is when we determine that CRT has failed. A positive biopsy may define the endpoint conclusively, but a premature positive biopsy may indicate active tumour, which is destined to disappear if the tumour is observed for a longer period.

\subsection{Recurrence-free Survival (RFS)}

RFS includes any recurrence (local, regional or distant) and also death due to any cause (both anal cancer and non-anal cancer causes of death). In the original ACT II protocol, a primary endpoint was recurrence-free survival (defined as first recurrence or death from any cause and also date of complete clinical response (CCR). When published we renamed this definition to progression-free survival (PFS).

\subsection{Progression free Survival (PFS)}

In ACT II we referred to PFS as the primary endpoint. The original ACT II protocol used the term 'RFS', at the time of publication this was considered misleading and PFS best suited the events included in the original endpoint definition. The events are captured in a noncontinuous framework, so timing/intervals between the clinical and radiological assessments are crucial to the precision of this date. PFS in metastatic disease is not defined by the stable persistence of tumour, but by enlargement or the appearance of new lesions. In contrast, in SCCA following CRT no observed change to the original primary tumour is eventually considered as progression. Hence PFS is ambiguous and a new term is required for patients treated with radical CRT with HNSCC, oesophageal cancer, cervix and SCCA. This term should accurately capture this event.

PFS can be defined as first clinical detection of disease progression (preferably defined by biopsy) (ie, local, regional or distant), recurrence or death from any cause, with censoring of 
the very few patients who are lost to follow-up or did not experience the event on their date last seen alive. This definition of PFS does not capture persistence of primary tumour. PFS can therefore be criticised as a primary endpoint because of the potential liability/ subjectivity which depends on the frequency and timing of radiographic surveillance. Subsequent lines of treatment or salvage surgery can affect OS. So information on subsequent treatment after documented progression is essential ${ }^{21}$. Discussion regarding PFS and its limitations ${ }^{22}$ is beyond the scope of this review.

\subsection{Local failure free survival (LFFS)}

In ACT I the primary endpoint in both the protocol and the 1996 report was defined as the occurrence of local failure ${ }^{9}$ which was a composite of local regional failure and the need for surgery for treatment-related morbidity, or 6 months after the end of treatment if a pretreatment colostomy had not been closed (assessed from 6 weeks after initial treatment). Local tumour failure was defined as evidence of persistent local disease, local regrowth or local recurrence in the primary tumour after protocol therapy. Patients who never attained local control (after chemoradiotherapy) were counted as treatment failures at the first assessment post-treatment at 6 weeks. This composite endpoint assumed all patients with persistent or recurrent disease would have a colostomy fashioned, which turned out not to be the case because $31 \%(82 / 265)$ of patients with local failure were too advanced, too frail or otherwise unsuitable for surgery, $8 \%$ (20/265) requiring colostomies for treatment morbidity and 5\% (14/265) unexplained failure to close a pre-treatment colostomy. By including all these events the trial described a population both tumour and colostomy-free, which was a useful comparison to surgery as primary therapy, but for contemporary trials separate data on disease and colostomy status is required.

Local failure-free-survival seems the most logical endpoint as it includes all relevant events, irrespective of surgical salvage and does not have the problems associated with DFS (not all patients are disease free at baseline) or PFS (persistent stable disease as an event). Yet, in the RTOG 8704 study patients who had a colostomy, abdominoperineal resection, or exenteration for any reason were considered treatment failures on the day of surgery - even if subsequent long-term local control was achieved $\mathbf{8}^{\mathbf{8}}$ Even if salvage surgery remains possible after locoregional failure the survival gain may be offset by permanent functional impairment, and a decreased quality of life, although many will accept these changes to survive. 


\subsection{Loco-regional failure free Survival (LRFS)}

Regional failure is defined by the RTOG as persistence, regrowth or recurrence of regional nodal disease and therefore loco-regional failure can be defined as clinically proven (preferably by biopsy) local failure or disease recurrence in pelvic lymph nodes included in the original external beam treatment volume, irrespective of any distant failures. Patients with persistent disease, who are never disease-free, are considered to be in failure on the day of randomisation or if disease disappears and then recurs - on the date of biopsy-proven or convincing imaging evidence of recurrent disease (when available). Salvage surgery for the primary site (unless histopathology showed no residual tumour) and death as a result of index cancer without a documented site of recurrence or unknown cause are considered LRF.

LRF and metastatic disease should be analyzed separately as the site of first failure. Since different doses are mandated for involved nodes and the primary tumour compared to elective nodes, it would probably also be important to separate/distinguish local primary failure and local regional failure within the treatment fields as separate endpoints, as well as locoregional failure outside the treatment fields. The 3-year rate of pelvic loco-regional disease related events - based on time-to-event analyses with censoring should be the defining factor.

\subsection{Second malignancy}

Second malignancies are common in SCCA. In ACT II 20 patients died of other cancers - in total 6 who received mitomycin C (MMC) based CRT and 14 who received cisplatinum either as CRT or maintenance ${ }^{2}$. Some investigators consider the development of SCCA in the anorectum after a disease-free interval of 3 to 5 years to be a new primary tumour, therefore any "local failure" after 3 to 5 years may be miscategorized and confound the analysis. Other studies consider $2^{\text {nd }}$ malignancy as contributing to DFS. Such decision-making should be made clear in the protocol, and separated from the key analyses.

\subsection{Colostomy-free survival (CFS)}

For patients being both disease, and colostomy-free, is important. However, only four of the six trials reported on this outcome. Both the ACT I and The European Organization for Research on Treatment of Cancer (EORTC) 22921 trial showed significant improvement in CFS in patients who received chemoradiation compared to radiotherapy ${ }^{\mathbf{1 0}, \mathbf{1 3}}$. CFS was the primary outcome measure of the ACCORD-03 trial, and a secondary endpoint in ACT II and RTOG 9811. 
All trials included colostomy formation as part of salvage surgery after local disease relapse, which fails to account for the need for colostomy in the absence of disease occurring either post-treatment for excessive faecal discharge/incontinence, or pre-treatment to avoid morbidity. Trials do not document whether subsequent reversal is or is not achieved. These non-disease colostomy events were included in the CFS analysis in the ACT I and II trials and although CFS captures both disease and treatment it is a poorly discriminating endpoint ${ }^{23}$. This is because the intervention will vary from unit to unit and so is subject to inherent selection bias. Pre-treatment colostomy is not part of the randomised allocation, some patients refuse to have a stoma for any reason, and there are well-recognised geographical and cultural differences in acceptance of a colostomy.

\section{Non-time-to-event Endpoints}

\subsection{Response}

Tumour response is the most commonly used indicator of antitumor activity, and can provide an objective assessment - given that cancers rarely shrink spontaneously ${ }^{\mathbf{2 4}}$. Overall tumour response has limitations as a surrogate endpoint for long-term clinical outcomes, but CCR is a valid endpoint if there is evidence that this can be sustained for long periods.

In SCCA, sustained CCR after definitive CRT is considered a useful early clinical endpoint, because CCR implies destruction of the cancer and possible avoidance of a permanent stoma. There is a balance between waiting for a response (a minimum of 4 weeks) versus the need for early salvage surgery before the tumour grows and becomes unresectable ${ }^{\mathbf{2 5}}$. Response to CRT has been assessed histopathologically, clinically and radiologically, with a less welldefined role for endoanal ultrasound and magnetic resonance imaging (MRI) ${ }^{26}$.

After CRT the interval to best response may be partially dependent on the tumour (size, stage or nodal status) or the modality of treatment (radiotherapy or chemoradiation). It is therefore clear that standardized serial clinical and imaging assessments are required for follow-up and the timing of CCR as an event should be defined eg. CCR 26 weeks from start of treatment ${ }^{25}$. Although standard RECIST criteria ${ }^{27}$ are applied, the RECIST system was not designed for primary tumour assessment, but stipulates assessment at 6-8 weeks and excludes tumours under $1 \mathrm{~cm}$. 


\subsection{Adverse events}

The Consolidated Standards of Reporting Trials (CONSORT) guidance offers specific and comprehensive guidelines regarding adverse event (AE) reporting in randomized clinical trials (RCTs), but adherence to these guidelines appears poor in oncology ${ }^{28}$. Also, the maximum adverse event grade may be less relevant than a progressive worsening of the adverse event over time.

\subsubsection{Acute toxicity}

Where overall survival is unlikely to be improved by a novel intervention (for example IMRT vs standard radiation), acute toxicity may be a suitable primary endpoint ${ }^{29}$. Different studies with varying types and intensities of chemotherapy, with a range of radiotherapy doses and schedules would be likely to lead to different toxicity profiles. Acute toxicity and compliance have very broad definitions, which include different symptoms and conditions and protocolmandated dose reductions. Defining toxicity is also important because the severity of acute effects has been correlated with eventual improved outcomes ${ }^{30}$.

Specific adverse events may be flagged as more important for a particular drug, with the causality and duration of the AE episode estimated. The number of patients experiencing these adverse events are recorded and distinguished by severity levels and according to treatment arm.

Toxicity assessments can be to some extent subjective between patient groups, measured using different assessment tools (WHO/NCI/CTC), and provide very different levels of compliance depending on the scale of the dose reductions for toxicity recommended. Therefore another possible explanation of the heterogeneity demonstrated for toxicity in these studies is that they are, indeed, reflecting different results. Furthermore, varying assessment periods, 4-8 weeks following completion of treatment, are used.

In the ACT 1 trial the toxicity scale used was simply "mild, moderate and severe" and graded subjectively by the investigator ${ }^{9}$. Although meaningful it is difficult to compare this scale with other more modern assessments. The EORTC used the World Health Organisation (WHO) 1979 acute morbidity scoring system, but the RTOG-8704 and the RTOG-9811 assessed chemotherapy toxicity according to the National Cancer Institute (NCI) common 
toxicity criteria (CTC), and RT toxicity was graded according to RTOG toxicity criteria for radiation effects ${ }^{\mathbf{8}, 11}$. whereas ACT II used the NCI CTC toxicity scale ${ }^{2}$.

The use of patient reported outcome measures PROMS is recommended ${ }^{31}$ because many important symptoms are so subjective and often poorly or under-categorised by clinicians ${ }^{\mathbf{3 2}}$. The number and manner of PROMS/QOL measures to be collected should be documented in the Patient Information Sheet (PIS) so that patients both understand what might be expected and are not worried that they may be questioned too often.

\subsubsection{Toxic deaths}

Specific definitions of treatment- or cancer -related mortality in an elderly patient population with multiple co-morbidities is problematic. Our experience is that defining death events as treatment-related is subjective. It may be better to report cause of death as anal cancer, treatment-related (including acute deaths such as neutropenic sepsis or myocardial infarction) or as non-cancer deaths. Alternatively, deaths within 90 days of commencing therapy could be documented separately.

\subsubsection{Late effects}

There is no common language and no standardised and well-defined current system developed for both recording and reporting acute and late radiation morbidity. Nor is there an accepted time frame - late is usually considered morbidity persistent/existing after 6 or 12 months, but in some studies is considered only after 5 years. Patients are poor at reporting symptoms ${ }^{\mathbf{3 3}}$ and whilst questionnaires can increase responses, these questions are designed to identify pre-morbidity as opposed to radiotherapy-associated effects. The current RTOG late effects instrument ${ }^{\mathbf{3 4}}$ is not sufficiently specific or extensive enough. Hence, appropriate PROMS are in development (see QOL).

\subsubsection{Tolerability}

Secondary tolerability endpoints could include:

(1) Dose intensity achieved (mg/m2/week): the total dose per body surface area divided by the duration of drug treatment (the number of weeks between start and finish of chemotherapy)

(2) The relative dose intensity (\%): ie the ratio of the dose intensity achieved compared to the planned dose intensity 
(3) The relative treatment duration: the ratio of the duration of treatment observed in the trial to the planned duration of treatment.

The reasons for reductions, delays, omissions should be documented to indicate whether due to toxicity or other cause.

\subsection{Compliance}

Compliance refers to the degree or extent of conformity to the trial recommendations with respect to timing, dosage, and frequency of intended treatment. Compliance should be distinguished from continuance of the treatment for the prescribed duration ${ }^{35}$.

Reporting compliance is essential for the interpretation of results and to inform the impact of the treatment when applied in a real world setting. Definitions vary. Without data on compliance reproducibility of trial results may not be possible. For example in the RTOG 9811 the definition of radiotherapy compliance was 'per protocol and an acceptable minimal variation' in radiation dose thereby categorising patients with less than total dose as compliers. Compliance in ACT II was defined as patients receiving full dose only. Clear descriptions of median radiation dose received and overall treatment time (OTT) with interquartile ranges are required. A simple composite classification of the adequacy of radical chemoradiation using three grades based on the actual drug doses received, the dose intensity and duration in days of any planned or unintended break in treatment may suffice.

Compliance to concurrent chemotherapy is also problematic as the chemotherapy dose will be compromised to ensure maximum radiotherapy dose if toxicity occurs. The second course of chemotherapy is crucial to maintain efficacy ${ }^{36}$. A conservative trial design allowing $50 \%$ dose-reductions for subsequent chemotherapy courses if grade 3 or above specific toxicities occur will have a lower dose intensity than less permissive protocols. A more useful summary might be to report both total dose and dose intensity curves ${ }^{37}$

\subsection{Patient Reported Outcomes (PROMS)}

Only two trials captured data on Quality of Life (QoL) due to the absence of available validated questionnaires specific to SCCA at the time of trial design. Using generic Rotterdam Symptom Checklist and Hospital Anxiety and Depression Scales chemoradiotherapy appeared to improve QOL compared to radiotherapy alone, but this was probably due to better disease control $^{\mathbf{3 8}}$. Some used the EORTC QLQ-C30 and Anal 
Sphincter Conservative Treatment Questionnaire short-term QOL in SCCA during treatment and shortly after to document $\mathrm{QOL}^{39}$. There are known adverse effects of pelvic radiotherapy on continence and quality of life ${ }^{\mathbf{4 0}}$. A mixed methods approach to PROMs for patients treated for SCCA identified gaps in the currently available questionnaires, and indicate that the EORTC-QLQ questionnaire is the most comprehensive in terms of symptom items ${ }^{\mathbf{4 1}}$. We therefore recommend including long-term reports of continence and/or QoL using PROMS in future trials.

\section{DISCUSSION}

An important limitation of this analysis is that it is based on only 6 randomized trials with different entry criteria and different treatments. As the efficacy of chemoradiotherapy improves in SCCA, and higher RT doses are integrated with more sophisticated irradiation techniques such as intensity modulated radiotherapy (IMRT), we may observe similar findings to head and neck cancer, where loco-regional control and overall survival is decoupled, because distant events after treatment are more common.

A systematic review of $125 \mathrm{RCT}$ s found clear definitions even of the survival endpoint were lacking in almost half these papers ${ }^{\mathbf{4 2}}$. Much effort has been expended on adjuvant endpoints following surgery and for metastatic disease, but there has been less focus on endpoints following radical treatment of loco-regional pelvic disease with chemoradiation. Neither the EORTC radiotherapy group ${ }^{\mathbf{4 3}}$ nor more recent aspirations designing phase III trials specifically addressed such endpoints ${ }^{44}$.

The choice of the most appropriate and unambiguous outcome measures is a vital component of trials as promoted by the (CONSORT) statement ${ }^{6}$. The utility of cross-trial comparisons and meta-analyses remain limited ${ }^{\mathbf{4 5}}$. Positive results can also sometimes represent a chance finding, or factors within an underpowered trial provide a heterogeneous patient population which confound the results ${ }^{46}$.

At baseline all patients prior to CRT have disease and the majority achieve a complete response, others despite initial response are never free from disease and either remain in this state, or have no detectable disease following salvage surgery. Current outcomes such as DFS and PFS can be difficult to apply in this situation as they are most clearly used when patients either have undetectable disease or measurable disease at the point of origin and are 
therefore all at risk of recurrence or progression. Also the tumour may initially respond, but the nadir may not be defined and the actual date of progression is never likely to be accurately defined but overestimated by the timing of the next scan or doctor visit.

Current trials in (SCCHN) use PFS and its components (LRF and DM), which are often reported instead of protocol-specified disease-free-survival to facilitate comparison with published meta-analyses ${ }^{47}$.

Usually 3-9 months following completion of treatment, if tumour is still present, we conclude that the patient has residual active cancer and chemoradiation has failed. Either radiologically the tumour enlarges or a steady persistence of disease at this arbitrary time-point becomes defined as progression. There is necessarily not a clear distinct line between disease and no disease, but it represents a dynamic process.

It is also well recognised that secondary endpoints are often defined and assessed less rigorously ${ }^{\mathbf{4 8}}$. The need to develop optimal primary and secondary endpoints for clinical trials, will become increasingly important as the trials get more complex. Improvements in trial design need to be accompanied by improvements in available endpoints and patients and investigators will need to work together to achieve this ${ }^{\mathbf{4 9}}$.

\section{CONCLUSION}

The objectivity, reliability, and validity of the current endpoints in clinical trials are variable. Time-to-event (TTE) endpoints other than OS share little uniformity across RCTs in SCCA. Different trials use different procedures to consider a patient as having an event which is not consistent with other studies. Rigorous definitions and consistent terminology are mandatory for future studies. The validity and feasibility of these endpoints for future international trials has already been discussed in IRCI meetings and we hope to work towards a consensus document.

We recommend consistency in reporting acute and late toxicity and compliance, and support the DATECAN project ${ }^{\mathbf{5 0}}$ for consensus-based recommendations. In rare cancers, unanimously agreed definitions are essential, because large long-term studies are few and difficult to perform. Journals in particular should agree to accept only standard definitions for 
survival endpoints. Hence investigators, statisticians, reviewers, and editors should all take responsibility for the precision of trial endpoints.

The ideal objective for gauging success in future SCCA phase III trials should be anal dysfunction-free survival. An internationally agreed definition should form the primary endpoint. We recommend the following secondary endpoints: OS and CSS as well as information on deaths not due to anal cancer. The late effects of radiotherapy captured by PROMS with long-term follow-up are essential. We also recommend the use of CFS and RFS - which includes any recurrence (local or regional, or distant) and also death due to any cause. Long-term follow-up for overall survival is still required in case unexpected adverse effects of treatment are not captured by this earlier endpoint. Yet, with the increasing development of more effective immunological treatments for metastatic disease, RFS may be less relevant and uncoupled from OS. Since most recurrences occur within the first 3 years, a minimum of 3 years monitoring and follow-up is mandatory for the required number of events to be captured.

Future randomised trials in SCCA should document the median/mean radiation dose received, and the compliance to chemotherapy during each week of treatment (as a percentage of the intended dose), the total dose of radiation achieved, the OTT, the precise site of recurrence in relation to radiotherapy fields.

Finally, we recommend that methodological research should address the validation of surrogate end-points, (such as local control/complete clinical response at 6 months).

\section{REFERENCES}

1. Wilson MK, Karakasis K, Oza AM. Outcomes and endpoints in trials of cancer treatment: the past, present, and future. Lancet Oncology 2015 Jan;16(1):e32-42.

2. James RD, Glynne-Jones R, Meadows HM, et al: Mitomycin or cisplatin chemoradiation with or without maintenance chemotherapy for treatment of squamous-cell carcinoma of the anus (ACT II): a randomised, phase 3, open-label, $2 \times 2$ factorial trial. Lancet Oncol 2013;14(6):516-24.

3. Sclafani F, Adams RA, Eng $\mathrm{C}$ et al. InterAACT: An international multicenter open label randomized phase II advanced anal cancer trial comparing cisplatin (CDDP) plus 5fluorouracil (5-FU) versus carboplatin (CBDCA) plus weekly paclitaxel (PTX) in patients with inoperable locally recurrent (ILR) or metastatic disease. J Clin Oncol 2015;33, (suppl 3; abstr TPS792). 
4. Michiels S, Le Maître A, Buyse M, et al. Surrogate endpoints for overall survival in locally advanced head and neck cancer: Meta-analyses of individual patient data. Lancet Oncol 2009; 10:341-350.

5. Food and Drug Administration H. International Conference on Harmonisation; Guidance on Statistical Principles for Clinical Trials; Availability. Fed Regist 1998; 63(179):49583-98.

6. Schulz K F, Altman D G, Moger D, (for the CONSORT group). CONSORT 2010 statement: updated guidelines for reporting parallel group randomised trials. Br Med J 2010; 340: c332.

7. Kilburn LS, Peckitt C, Ireland E, et al: Defining endpoints for recurrence in randomized controlled trials of systemic therapy for early breast cancer: A call for standardization. San Antonio Breast Cancer conference; 2007December 13-16, San Antonio, TX. (abstr 6035).

8. Flam M, John M, Pajak TF, et al: Role of mitomycin in combination with Fluorouracil and radiotherapy, and of salvage chemoradiation in the definitive nonsurgical treatment of epidermoid carcinoma of the anal canal: Results of a phase III randomized Intergroup Study. J Clin Oncol 1996;14:2527-2539.

9. UKCCCR Anal Cancer Working Party. Epidermoid Anal Cancer: Results from the UKCCCR randomised trial of radiotherapy alone versus radiotherapy, 5-fluorouracil and Mitomycin. Lancet 1996;348:1049-1054.

10. Bartelink H, Roelofsen F, Eschwege F, et al: Concomitant Radiotherapy and Chemotherapy Is Superior to Radiotherapy Alone in the Treatment of Locally Advanced Anal Cancer: Results of a Phase III Randomized Trial of the European Organization for Research and Treatment of Cancer Radiotherapy and Gastrointestinal Cooperative Groups. J Clin Oncol 1997; 15:2040-2049.

11. Ajani JA, Winter KA, Gunderson LL, et al: Fluorouracil, mitomycin and radiotherapy vs fluorouracil, cisplatin and radiotherapy for carcinoma of the anal canal: a randomised controlled trial. JAMA 2008;199:1914-21.

12. Peiffert D, Tournier-Rangeard L, Gérard JP, et al: Induction Chemotherapy and Dose Intensification of the Radiation Boost in Locally Advanced Anal Canal Carcinoma: Final Analysis of the Randomized UNICANCER ACCORD 03 Trial. J Clin Oncol 2012; Jun 1; 30(16):1941-8.

13. Northover JMA, Glynne-Jones R, Sebag-Montefiore D, et al: Chemoradiation for the treatment of epidermoid anal cancer: 13-year follow-up of the first randomised UKCCCR Anal Cancer Trial (ACT I). Br J Cancer 2010 Mar 30; 102:1123-8.

14. Gunderson LL, Winter KA, Ajani JA, et al: Long-term update of U.S. GI Intergroup RTOG 98-11 phase III trial for anal carcinoma: Survival, Relapse and Colostomy failure with concurrent chemoradiation involving Fluorouracil/mitomycin versus Fluoruracil/cisplatin. J Clin Oncol 2012; 30:4344-51. 
15. Gunderson LL, Moughan J, Ajani JA et al. Anal carcinoma: impact of TN category of disease on survival, disease relapse, and colostomy failure in US Gastrointestinal Intergroup RTOG 98-11 phase 3 trial. Int J Radiat Oncol Biol Phys. 2013 Nov 15;87(4):638-45.

16. Pintilie M. Competing Risks: A Practical Perspective. New York: Wiley; 2006.

17. Mariani P, Ghanneme A, De la Rochefordière A, Girodet J, Falcou MC, Salmon RJ. Abdominoperineal resection for anal cancer Dis Colon Rectum. 2008;51(10):1495 -501.

18. Lefèvre JH, Corte $\mathrm{H}$, Tiret $\mathrm{E}$ et al. Abdominoperineal resection for squamous cell anal carcinoma: survival and risk factors for recurrence. Ann Surg Oncol. 2012 Dec;19(13):418692.

19. Takashima A, Shimada Y, Hamaguchi T et al; Colorectal Cancer Study Group of the Japan Clinical Oncology Group. A Phase I/II trial of chemoradiotherapy concurrent with S-1 plus mitomycin $\mathrm{C}$ in patients with clinical Stage II/III squamous cell carcinoma of anal canal (JCOG0903: SMART-AC). Jpn J Clin Oncol. 2011 May;41(5):713-7.

20. Robinson AG, Booth CM, Eisenhauer EA. Disease-free survival as an end-point in the treatment of solid tumours--perspectives from clinical trials and clinical practice. Eur $\mathbf{J}$ Cancer. 2014 Sep;50(13):2298-30.

21. Robinson AG, Booth CM, Eisenhauer EA. Progression-free survival as an end-point in solid tumours--perspectives from clinical trials and clinical practice. Eur J Cancer. 2014 Sep;50(13):2303-8.

22. Venook AP, Tabernero J. Progression-free survival: helpful biomarker or clinically meaningless end point? J Clin Oncol. 2015 Jan 1;33(1):4-6.

23. Glynne-Jones R, Kadalayil L, Meadows HM et al; Title of paper ACT II Study Group. Ann Oncol. 2014 Aug;25(8):1616-22.

24. Karnofsky DA. Meaningful clinical classification of therapeutic responses to anticancer drugs.Clin Pharmac Ther 1961; 2: 709-712.

25. Glynne-Jones R, James R, Meadows H, et al: Optimum time to assess complete clinical response (CR) following chemoradiation (CRT) using mitomycin (MMC) or cisplatin (CisP), with or without maintenance $\mathrm{CisP} / 5 \mathrm{FU}$ in squamous cell carcinoma of the anus: Results of ACT II. J Clin Oncol 2012;30 (suppl; abstr 4004).

26. Parikh J, Shaw A, Grant LA, Schizas AM et al: Anal carcinomas: the role of endoanal ultrasound and magnetic resonance imaging in staging, response evaluation and follow-up. Eur Radiol 2011, 21(4):776-785.

27. Eisenhauer EA, Therasse P, Bogaerts J, et al. New response evaluation criteria in solid tumours: revised RECIST guideline (version 1.1). Eur J Cancer 2009; 45: 228-47.

28. Péron J, Maillet D, Gan HK et al. Adherence to CONSORT adverse event reporting guidelines in randomized clinical trials evaluating systemic cancer therapy: a systematic review. J Clin Oncol. 2013 Nov 1;31(31):3957-63. 
29. Kachnic LA, Winter K, Myerson RJ, et al: RTOG 0529: A Phase 2 Evaluation of Dose-Painted Intensity Modulated Radiation Therapy in Combination with 5-Fluorouracil and Mitomycin-C for the Reduction of Acute Morbidity in Carcinoma of the Anal Canal. Int J Radi0t Oncol Biol Phys 2013;May 1;86(1):27-3.

30. Heemsbergen WD, Peeters ST, Koper PC. et al. Acute and late gastrointestinal toxicity after radiotherapy in prostate cancer patients: consequential late damage. Int J Radiat Onc1l Biol Phys. 2006;66(1):3-10.

31. Basch E, Reeve BB, Mitchell SA et al. Development of the National Cancer Insitute's patient reported outcomes version of the common terminology criteria for adverse events (PRO-CTCAE). J Natl Cancer Inst. 2014 Sep 29;106(9).

32, Di Maio M, Gallo C, Leighl NB, et al., Symptomatic toxicities experienced during anticancer treatment: agreement between patient and physician reporting in three randomized trials. J Clin Oncol. 2015 Mar 10;33(8):910-5.

33. Andreyev HJN, Davidson SE, Gillespie C, et al: Practice guidance on the management of acute and chronic gastrointestinal problems arising as a result of treatment for cancer. Gut 2012; 61(2):179-192.

34. Cox JD, Stetz J, Pajak TF: Toxicity criteria of the Radiation Therapy Oncology Group (RTOG) and the European Organization for Research and Treatment of Cancer (EORTC). Int J Radiat Oncol Biol Phys 31(5):1341-6, 1995

35. Cramer JA, Roy A, Burrell A et al. Medication compliance and persistence: terminology and definitions. Value Health. 2008 Jan-Feb;11(1):44-47.

36. Glynne-Jones R, Meadows AH, Lopes A et al. Compliance to chemoradiation (CRT) using mitomycin (MMC) or cisplatin (CisP), with or without maintenance 5FU/CisP chemotherapy (CT) in squamous cell carcinoma of the anus (SCCA) according to radiotherapy (RT) dose, overall treatment time (OTT) and chemotherapy (CT) and their impact on long-term outcome: Results of ACT II. J Clin Oncol 33, 2015 (suppl; abstr 3518).

37. The ICON Collaborators: ICON2: randomised trial of single-agent carboplatin against three-drug combination of CAP (cyclophosphamide, doxorubicin, and cisplatin) in women with ovarian cancer. The Lancet 1998;352:9140:1571-6.

38. Slevin ML, Plowman PN, Ryan CM et al., Chemoradiotherapy for anal cancer improves quality of life compared to radiotherapy alone. J Clin Oncol 1998;17:(abstract 266).

39. Tournier-Rangeard L, Mercier M, Peiffert D, et al. Radiochemotherapy of locally advanced anal canal carcinoma: prospective assessment of early impact on the quality of life (randomized trial ACCORD 03). Radiother Oncol. 2008 ;87(3):391-7.

40. Bentzen AG, Balteskard L, Wanderås EH, et al: Impaired health-related quality of life after chemoradiotherapy for anal cancer: late effects in a national cohort of 128 survivors. Acta Oncol 2013;52(4):736-44.

41. Gilbert A, Francischetto EO, Blazeby $\mathrm{J}$ et al. Choice of a patient-reported outcome measure for patients with anal cancer for use in cancer clinical trials and routine clinical practice: a mixed methods approach. Lancet. 2015;385 Suppl 1:S38. 
42. Mathoulin-Pelissier S, Gourgou-Bourgade S, Bonnetain F et al. Survival end point reporting in randomized cancer clinical trials: a review of major journals. J Clin Oncol 2008 Aug 1; 26(22):3721-6.

43. Bolla M, Bartelink $\mathrm{H}$, Garavaglia $\mathrm{G}$ et al. EORTC guidelines for writing protocols for clinical trials of radiotherapy. Radiother Oncol. $1995 \mathrm{Jul} ; 36(1): 1-8$.

44. Fairchild A, Bar-Deroma R, Collette L et al. Development of clinical trial protocols involving advanced radiation therapy techniques: the European Organisation for Research and Treatment of Cancer Radiation Oncology Group approach. Eur J Cancer. 2012 May;48(7):1048-54.

45. Kirkham J J, Dwan K M, Altman D G et al. The impact of outcome reporting bias in randomized controlled trials on a cohort of systematic reviews. Br Med J 2010;340:c365.

46. Blair E. Gold is not always good enough: the shortcomings of randomization when evaluating interventions in small heterogeneous samples. J Clin Epidemiol.

2004;57(12):1219-22.

47. Ang KK, Zhang Q, Rosenthal DI et al. Randomized phase III trial of concurrent accelerated radiation plus cisplatin with or without cetuximab for stage III to IV head and neck carcinoma: RTOG 0522. J Clin Oncol 2014;32(27);2940-50.

48. Matthews JH, Bhanderi S, Chapman SJ, Nepogodiev D, Pinkney T, Bhangu A. Underreporting of Secondary Endpoints in Randomized Trials: Cross-sectional, Observational Study.Ann Surg. 2016 Jan 7. [Epub ahead of print]

49. Wilson MK, Collyar D, Chingos DT, Friedlander M, Ho TW, Karakasis K,et al., Outcomes and endpoints in cancer trials: bridging the divide. Lancet Oncol. 2015 Jan;16(1):e43-52.

50. Bellera CA, Pulido M, Gourgou S et al: Protocol of the Definition for the Assessment of Time-to-event Endpoints in CANcer trials (DATECAN) project: Formal consensus method for the development of guidelines for standardised time-to-event endpoints' definitions in cancer clinical trials. Eur J Cancer 2013;49(4):769-81. 
Table 1. Primary and secondary endpoints in Randomised trials

\begin{tabular}{|c|c|c|}
\hline Trial & Primary Endpoint & Secondary Endpoints \\
\hline ACT 16,16 & $\begin{array}{l}\text { Local treatment failure (composite of } \\
\text { local failure and need for colostomy } \\
\text { for toxicity) }\end{array}$ & Overall survival \\
\hline EORTC $^{7}$ & Local failure & Event-free survival \\
\hline RTOG 87048 & Disease-free survival. & $\begin{array}{l}\text { Overall survival } \\
\text { Colostomy-free survival, } \\
\text { Time to colostomy } \\
\text { Loco-regional control, } \\
\text { Incidence of negative post induction biopsy } \\
\text { Incidence of positive salvage biopsy, } \\
\text { Toxicity rates) }\end{array}$ \\
\hline RTOG 98114 & Disease-free survival. & $\begin{array}{l}\text { Overall Survival } \\
\text { Cumulative incidence of Colostomy } \\
\text { Cumulative Incidence of Local Regional } \\
\text { Failure and Distant Metastases; } \\
\text { Toxicity } \\
\text { Hazard ratios for tumor markers P53 } \\
\text { overexpression, human papilloma virus } \\
\text { status and enzyme marker HAP1. }\end{array}$ \\
\hline $\operatorname{ACCORD~03^{5}}$ & Colostomy-free survival & $\begin{array}{l}\text { Overall survival } \\
\text { Cancer-specific survival } \\
\text { Local control }\end{array}$ \\
\hline ACT II & $\begin{array}{l}\text { (2 separate endpoints for } 2 \times 2 \\
\text { factorial design) Recurrence-free } \\
\text { survival } \\
\text { Complete response (complete } \\
\text { disappearance of clinically and } \\
\text { radiologically overt disease) } \\
\text { and acute toxicity: grade } 3 / 4 \mathrm{t} \text { up to } 4 \\
\text { weeks post-chemo for } \mathrm{MMC} / \mathrm{CisP} \\
\text { comparison }\end{array}$ & $\begin{array}{l}\text { Overall survival } \\
\text { Cancer-specific survival } \\
\text { Colostomy rate } \\
\text { In-field recurrence rate }\end{array}$ \\
\hline
\end{tabular}


Table 2: Definition of composite disease-related endpoints used in Anal Cancer Trials

\begin{tabular}{|c|c|c|c|c|c|c|c|}
\hline Trial & Endpoint & $\begin{array}{l}\text { Loco-regional } \\
\text { disease }^{\wedge}\end{array}$ & $\begin{array}{l}\text { Pelvic } \\
\text { disease }^{\wedge \wedge}\end{array}$ & $\begin{array}{l}\text { Distant } \\
\text { metastases }\end{array}$ & Death & New tumour & $\begin{array}{l}\text { Colostomy } \\
\wedge \wedge \wedge\end{array}$ \\
\hline ACT I ${ }^{1}$ & Local Treatment Failure & $\checkmark$ & $\checkmark$ & $\checkmark$ & $\checkmark$ & & $\checkmark$ \\
\hline ACT II ${ }^{6}$ & Progression-Free Survival* & $\checkmark$ & $\checkmark$ & $\checkmark$ & $\checkmark$ & & $\checkmark$ \\
\hline EORTC $^{7}$ & Event-Free Survival & $\checkmark$ & & & $\checkmark$ & $\checkmark$ & \\
\hline ACCORD 03 5 & Event-Free Survival & $\checkmark$ & & $\checkmark$ & $\checkmark$ & & \\
\hline $\begin{array}{l}\text { RTOG } \\
\text { 8704 }^{8} \\
\end{array}$ & Disease-Free Survival & $\checkmark$ & & $\checkmark$ & $\checkmark$ & & \\
\hline RTOG $9811^{4}$ & Disease-Free Survival & $\checkmark$ & & $\checkmark$ & $\checkmark$ & & \\
\hline
\end{tabular}

*definition for RFS defined in protocol used but renamed PFS

$\wedge$ locoregional disease includes original site and associated lymph nodes

$\wedge \wedge$ pelvic disease includes other pelvic organs and lymph nodes within the pelvis

$\wedge \wedge \wedge$ colostomy for treatment morbidity in absence of disease 
Table 3: Time-to-event endpoints used in Anal Cancer Trials

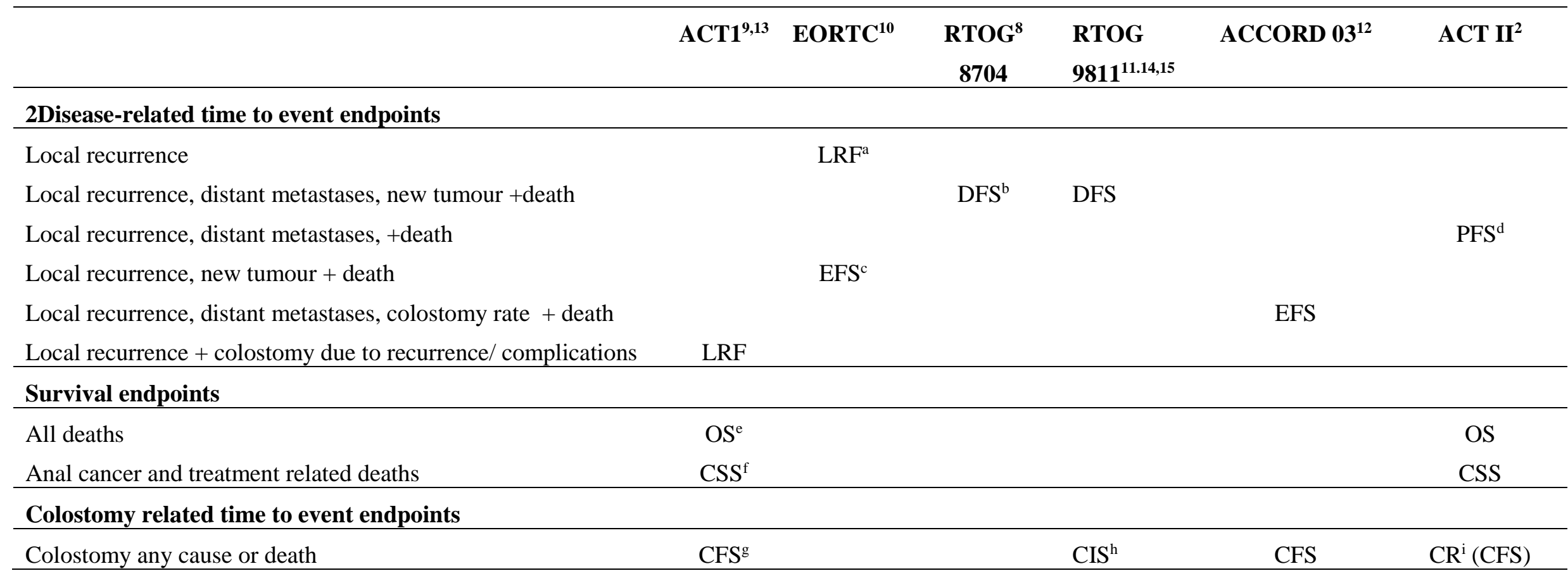

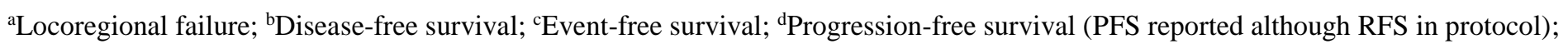

${ }^{\mathrm{e}}$ Overall survival; ${ }^{\mathrm{f}}$ Cause-specific survival

${ }^{\mathrm{g}}$ Colostomy-free survival ${ }^{10}$; ${ }^{\mathrm{h}} \mathrm{Cumulative} \mathrm{incidence} \mathrm{of} \mathrm{colostomy;}{ }^{\mathrm{i} C o l o s t o m y}$ rate 
Table 4 pros and cons of currently used endpoints

\begin{tabular}{|c|c|c|c|}
\hline Endpoint & Utility & Pros & Cons \\
\hline $\begin{array}{l}\text { Overall } \\
\text { Survival }\end{array}$ & Gold standard endpoint in randomised trials & $\begin{array}{l}\text { Easy to define, precise } \\
\text { universally accepted } \\
\text { available via registries }\end{array}$ & $\begin{array}{l}\text { Less robust as patient benefit if older } \\
\text { population and if surgery can salvage }>50 \%\end{array}$ \\
\hline $\begin{array}{l}\text { Cause-Specific } \\
\text { Survival }\end{array}$ & $\begin{array}{l}\text { Cause-specific survival focuses on the impact of the } \\
\text { cancer on survival. Competing events are treated as } \\
\text { censoring events, and death from causes unrelated to } \\
\text { carcinoma is considered lost to follow-up as of date of } \\
\text { death. Cause-specific survival analysis minimises the } \\
\text { impact of age, co-morbidity and other risk factors on } \\
\text { survival rates. }\end{array}$ & $\begin{array}{l}\text { Easy to define, widely } \\
\text { accepted. Useful in a cancer } \\
\text { which affects elderly patients, } \\
\text { and has effective surgical } \\
\text { salvage. }\end{array}$ & $\begin{array}{l}\text { Reliable information on the cause of death is } \\
\text { not always available. Death certificates are } \\
\text { often inaccurately reported. }\end{array}$ \\
\hline $\begin{array}{l}\text { Disease Free } \\
\text { Survival }\end{array}$ & $\begin{array}{l}\text { Often used post-surgery when no detectable disease } \\
\text { present at randomisation. Difficult to use in CRT trials } \\
\text { as proportion never disease free So ?censored }\end{array}$ & $\begin{array}{l}\text { Earlier endpoint than OS } \\
\text { Requires fewer numbers and } \\
\text { shorter follow-up }\end{array}$ & $\begin{array}{l}\text { Not validated as surrogate for survival in anal } \\
\text { cancer. Definitions vary between trials. Can } \\
\text { depend on frequency of imaging }\end{array}$ \\
\hline $\begin{array}{l}\text { Relapse/ } \\
\text { recurrence } \\
\text { Free Survival }\end{array}$ & $\begin{array}{l}\text { Used as primary endpoint when no detectable disease } \\
\text { present at trial entry. Difficult to use in CRT trials as } \\
\text { many never disease free So censored }\end{array}$ & $\begin{array}{l}\text { Earlier endpoint than OS } \\
\text { Requires fewer numbers and } \\
\text { shorter follow-up }\end{array}$ & $\begin{array}{l}\text { Subject to assessment bias } \\
\text { Can depend on frequency of imaging }\end{array}$ \\
\hline $\begin{array}{l}\text { Progression } \\
\text { Free Survival }\end{array}$ & $\begin{array}{l}\text { Often used in metastatic setting when all patients have } \\
\text { disease at randomisation. Used as primary endpoint in } \\
\text { trials }\end{array}$ & $\begin{array}{l}\text { Objective/quantitative } \\
\text { Not affected by salvage } \\
\text { surgery with APER or } \\
\text { subsequent treatment }\end{array}$ & $\begin{array}{l}\text { Stable disease not necessarily of clinical } \\
\text { benefit } \\
\text { Subject to assessment bias }\end{array}$ \\
\hline
\end{tabular}




\begin{tabular}{|l|l|l|l|}
\hline $\begin{array}{l}\text { Colostomy- } \\
\text { Free Survival }\end{array}$ & Used as primary endpoint in trials & Easy to define & $\begin{array}{l}\text { Initial colostomy can be reversed but often not } \\
\text { reversed } \\
\text { Colostomy can be formed both for recurrence } \\
\text { and late effects }\end{array}$ \\
\hline $\begin{array}{l}\text { Complete } \\
\text { clinical } \\
\text { response }\end{array}$ & $\begin{array}{l}\text { Often used as a surrogate endpoint especially in phase } \\
\text { II and phase III trials }\end{array}$ & $\begin{array}{l}\text { Assessed early (6 months) } \\
\text { smaller studies possible }\end{array}$ & $\begin{array}{l}\text { Imperfect } \\
\text { Needs to be sustained for clinical benefit } \\
\text { Not direct measure of clinical benefit Time } \\
\text { dependent. Prone to immortal time bias }\end{array}$ \\
\hline
\end{tabular}


Online Table 1. Complete list of search terms applied

\begin{tabular}{|c|c|}
\hline Area & Terms \\
\hline Anal Cancer & $\begin{array}{l}\text { Anus neoplasm (MeSH term) } \\
\text { Anal neoplasm } \\
\text { Anal cancer } \\
\text { Anus cancer } \\
\text { Anal carcinoma } \\
\text { Anus carcinoma (no hits) } \\
\text { Anal canal cancer } \\
\text { Anal canal carcinoma } \\
\text { Anal tumour } \\
\text { Anus tumour (no hits) } \\
\text { Anal intraepithelial neoplasia } \\
\text { Anal canal intraepithelial neoplasia } \\
\text { Anal squamous intraepithelial lesions } \\
\text { Anal squamous cell carcinoma } \\
\text { Anal cloacogenic carcinoma (no hits) } \\
\text { Cloacogenic carcinoma of the anal canal }\end{array}$ \\
\hline $\begin{array}{l}\text { Treatments } \\
\text { Radiochemotherapy } \\
\text { Stoma }\end{array}$ & $\begin{array}{l}\text { Chemoradiotherapy } \\
\text { Radiochemotherapy } \\
\text { Chemoradiation } \\
\text { Chemotherapy } \\
\text { Radiotherapy } \\
\text { Combined modality therapy } \\
\text { Antineoplastic chemotherapy } \\
\text { Antineoplastic agents } \\
\text { Colostomy } \\
\text { Surgical stoma (Exp Stoma and stoma bag) }\end{array}$ \\
\hline Health-related quality of life & $\begin{array}{l}\text { Quality of Life } \\
\text { QOL } \\
\text { Health related quality of life } \\
\text { HRQOL } \\
\text { Subjective health status } \\
\text { Patient reported outcome } \\
\text { Patient based outcome } \\
\text { Patient reported outcome measure } \\
\text { PROM } \\
\text { Self report } \\
\text { Side effect } \\
\text { Toxicity } \\
\text { Adverse effect } \\
\text { Adverse event } \\
\text { Safety } \\
\text { Complication } \\
\text { Dysfunction } \\
\text { Disturbance } \\
\text { Disorder } \\
\text { Impairment } \\
\text { Complaint } \\
\text { Symptom }\end{array}$ \\
\hline
\end{tabular}


Table 2: Showing important compliance to RT parameters

\begin{tabular}{|l|l|l|l|}
\hline Mean Dose of RT received & Median & Range & \\
\hline $\begin{array}{l}\text { \% of patients receiving 90-110\% of total } \\
\text { dose recommended }\end{array}$ & Median & Range & \\
\hline Number of days RT omitted & Median & Range & Reasons \\
\hline Number of days RT dose reduced & Median & Range & Reasons \\
\hline Overall treatment time (OTT) in days & Median & Range & Reasons \\
\hline
\end{tabular}

Table 3: Showing important compliance to chemotherapy parameters

\begin{tabular}{|l|l|l|l|}
\hline Mean Dose of chemotherapy received & Median & Range & \\
\hline $\begin{array}{l}\text { \% of patients receiving 90-110\% of total } \\
\text { dose recommended }\end{array}$ & Median & Range & \\
\hline Number of days chemotherapy omitted & Median & Range & Reasons \\
\hline $\begin{array}{l}\text { Number of days chemotherapy dose } \\
\text { reduced }\end{array}$ & Median & Range & Reasons \\
\hline $\begin{array}{l}\text { If delay in administration - Overall } \\
\text { treatment time (OTT) in days }\end{array}$ & Median & Range & Reasons \\
\hline
\end{tabular}

Figure 1. Time-to-event outcomes in SCCA - possible outcomes for patients with SCCA following chemoradiation.

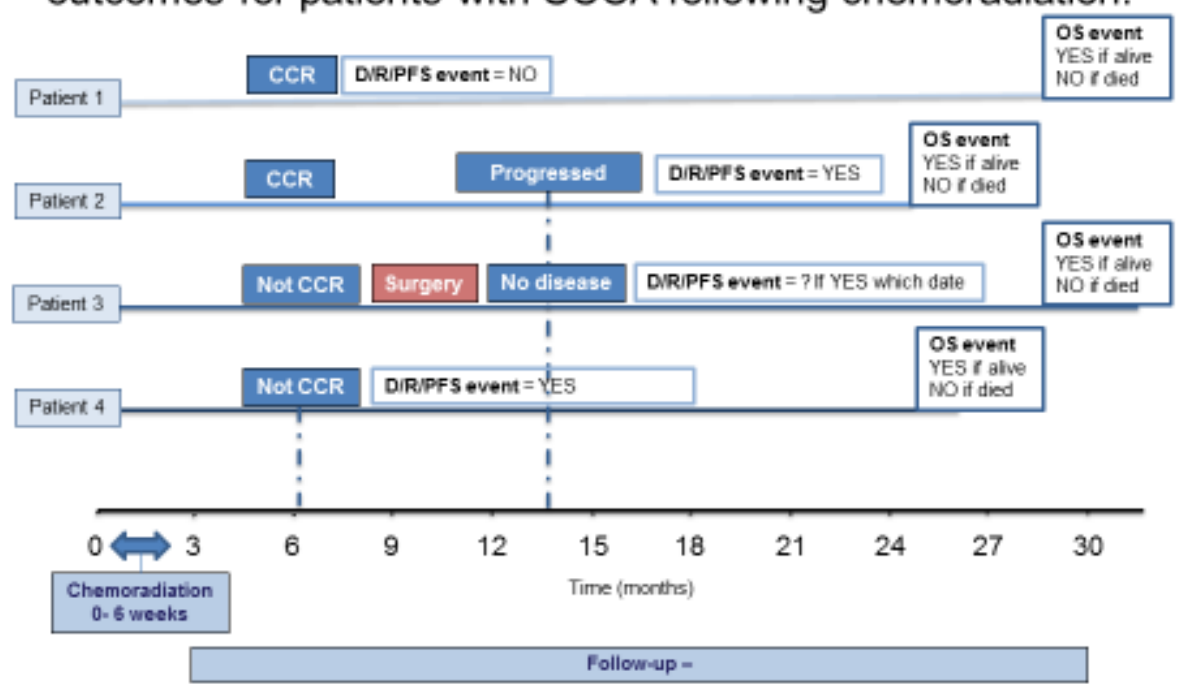

